Research Paper

\title{
Dynamic Contrast-Enhanced MR Imaging in a Phase II Study on Neoadjuvant Chemotherapy Combining Rh-Endostatin with Docetaxel and Epirubicin for Locally Advanced Breast Cancer
}

\author{
Qianxin Jia ${ }^{1,2^{*}}$, Junqing $\mathrm{Xu}^{1^{*}}$, Weifeng Jiang ${ }^{3}$, Minwen Zheng, ${ }^{1}$ Mengqi Wei ${ }^{1}$, Jianghao Chen ${ }^{4}$, Ling Wang4, \\ Yi Huan ${ }^{1 凶}$ \\ 1. Department of Radiology, Xijing Hospital, Fourth Military Medical University. 17 Changle Western Road, Xi' an, China. \\ 2. Department of Radiology, Dongnan Hospital, Xiamen University. 269 Zhanghua Middle Road, Zhangzhou, China. \\ 3. Department of Rehabilitation, Guangzhou Sanatorium of Guangzhou Military Region.1849 Guangzhou Avenue, Guangzhou, China. \\ 4. Department of General Surgery, Xijing Hospital, Fourth Military Medical University. 17 Changle Western Road, Xi'an, China. \\ * Equal study contribution.
}

$\triangle$ Corresponding author: Yi Huan, Department of Radiology, Xijing Hospital, Fourth Military Medical University. 17 Changle Western Road, Xi'an, China; E-mail: jiaqianxin@vip.sina.com, Tel: +86 29 84775421; Fax: +86 2984775421.

() Ivyspring International Publisher. This is an open-access article distributed under the terms of the Creative Commons License (http://creativecommons.org/ licenses/by-nc-nd/3.0/). Reproduction is permitted for personal, noncommercial use, provided that the article is in whole, unmodified, and properly cited.

Received: 2012.08.28; Accepted: 2012.12.26; Published: 20I2.12.28

\begin{abstract}
Background: Anti-angiogenesis is a promising therapeutic strategy for locally advanced breast cancer. We performed this phase II trial to evaluate the anti-angiogenesis and anti-tumor effect of rh-endostatin combined with docetaxel and epirubicin in patients with locally advanced breast cancer by dynamic contrast-enhanced magnetic resonance imaging in 70 previously untreated locally advanced breast cancer patients.

Methods: The study population was randomly assigned to neoadjuvant chemotherapy with docetaxel and epirubicin (neoadjuvant chemotherapy group) or neoadjuvant chemotherapy combining rh-endostatin with docetaxel and epirubicin (neoadjuvant chemotherapy+rh-endostatin group). The anti-angiogenic and anti-tumor effects of both regimens were evaluated by serial dynamic contrast-enhanced magnetic resonance imaging and microvessel density measurements after final surgery.

Results: The results suggested a higher clinical objective response $(90.9 \%$ vs. $67.7 \%, P=0.02 \mathrm{I})$ and greater reductions in tumor size $(67.2 \%$ vs. $55.9 \%, \mathrm{P}=0.000)$, Ki-67 proliferation index $(32.79 \%$ vs. $12.47 \%, P=0.000)$, tumor signal enhanced ratio $(64 \%$ vs. $48 \%, P=0.018)$, and $K^{\text {trans }}(67 \%$ vs. $39 \%$, $P=0.026$ ) in neoadjuvant chemotherapy+rh-endostatin group than those in neoadjuvant chemotherapy group. In addition, the microvessel density value in the neoadjuvant chemotherapy+rh-endostatin group was significantly lower than in the neoadjuvant chemotherapy group ( $18.67 \pm 6.53$ vs. $36.05 \pm 9.64, P=0.000)$. Moreover, the microvessel density value was significantly correlated with $\mathrm{K}^{\text {trans }}$ after neoadjuvant chemotherapy+rh-endostatin treatment $(r=0.88, P=0.00)$.

Conclusions: The neoadjuvant chemotherapy+rh-endostatin treatment significantly repressed angiogenesis in locally advanced breast cancer and synergistically enhanced the anti-tumor effect of neoadjuvant chemotherapy. Serial dynamic contrast-enhanced magnetic resonance imaging data including reductions in tumor size and $\mathrm{K}^{\text {trans }}$, could provide non-invasive evaluation for chemotherapeutic efficacy and, consequently, optimization of individual chemotherapy for locally advanced breast cancer patients.
\end{abstract}


Key words: breast cancer; neoadjuvant chemotherapy; rh-endostatin; microvessel density; dynamic contrast-enhanced MR imaging

\section{Introduction}

Neoadjuvant chemotherapy (NACT) has become a promising treatment for breast cancer, especially for locally advanced breast cancer. NACT may degrade the pathological stage of locally advanced breast cancer, decrease tumor size, and render the tumor resectable. In addition, previous studies suggest that the pathological response of primary locally advanced breast cancer to NACT can serve as a prognostic marker for patient outcome[1]. Therefore, ongoing studies are being conducted to develop novel NACT schemes and investigate their clinical efficacies by pathological examination[2].

Anti-angiogenesis is a novel frontier for the treatment of malignant disease given that tumor growth predominantly depends on its angiogenesis status[3]. Cell experiments and animal models have demonstrated the anti-angiogenesis and anti-tumor effects of dozens of angiogenesis inhibitors, among which is endostatin[4]. Endostatin, a $20 \mathrm{kDa}$ C-terminal fragment of collagen XVIII, is well known as a broad-spectrum, anti-angiogenic agent that specifically inhibits vascular endothelial cell proliferation, thereby inhibiting tumor angiogenesis and growth[5]. Endostatin has been demonstrated to be effective against different malignances, including breast cancer[6]. Recombinant human endostatin (rh-endostatin), which was purified from Escherichia coli and reconstructed with an additional nine-amino acid sequence thus to form another his-tag structure, has been shown to be at least twice as potent as endostatin in animal tumor models[7]. As shown in the previous studies, the underlying mechanisms by which rh-endostatin exerts significant anti-cancer bioactivity include: (i) prevention of the formation of tumor blood vessels through suppressing the VEGFR-2 expression in endothelial precursor cells; and (ii) obstruction of distant metastasis of breast cancer cells through inhibiting the intracellular expressions of MMP-2 and MMP-9[8]. A recent phase I study of rh-endostatin confirmed that endostatin, which was suggested to be well tolerated and essentially free of significant drug-related toxicity, provided clinical benefit with significant anti-angiogenesis and anti-tumor effects in advanced solid tumors such as non-small-cell lung cancer [9].Currently, rh-endostatin is approved for cancer therapy by the state food and drug administration of China.

Pathological examination by assessing microvessel density (MVD), which is principally based on surgical specimens, is currently the standard technique for determining tumor vascularity, one of the most important parameters for evaluating the in vivo effects of anti-angiogenic agents[10]. However, MVD is an invasive method and is therefore not suitable for serial study. Dynamic contrast-enhanced (DCE-MRI) is proven to be a noninvasive imaging technique that can be used to derive microcirculation properties[11]. Unlike MVD measurement, DCE-MRI can provide information on whole tumor volumes and not only on focal regions, which is more informative because tumor vasculature is heterogeneously distributed throughout the tumor[12]. Using a pharmacokinetic analysis, DCE-MRI can yield two parameters: the tumor transfer coefficient $\left(\mathrm{K}^{\text {trans }}\right)$ and the extravascular extracellular volume $\left(\mathrm{v}_{\mathrm{e}}\right)$, which can be used to evaluate tumor microvessel perfusion and the permeability of the tumor vasculature[13].

Little is known about the clinical efficacy of rh-endostatin for locally advanced breast cancer although its efficacy has been demonstrated in non-small cell lung cancers[14]. Therefore, in the current phase II study, we evaluated the anti-angiogenesis and anti-tumor effects of neoadjuvant treatment with rh-endostatin in combination with docetaxel and epirubicin in locally advanced breast cancer patients and tumor response using serial DCE-MRI.

\section{Patients and methods}

This open-labeled, prospective, randomized, phase II trial was approved by the local ethical committee of our institution and was approved by the Food and Drug Administration (Clinical trials.gov identifier: NACT00604435). From February 2008 to March 2010, a total of 70 female patients with previously untreated breast cancer were enrolled in the study. All patients were asked to provide written informed consent before enrollment.

\section{Patient eligibility}

Patient eligibility criteria included (i) cytopathologically confirmed locally advanced breast cancer (core needle biopsy for breast cancer diagnosis and fine needle aspiration for lymph node metastasis diagnosis); (ii) staged II A to III C; (iii) aged between 18 and 70 years; (iv) primary tumor size $\geq 1 \mathrm{~cm}^{3}$ based on ultrasonography; (v) Eastern Cooperative Oncology Group performance status 0 to 2; (vi) left ventricular ejection fraction greater than $50 \%$ without clinical 
symptoms or signs of heart failure; and (vii) no abnormality of organ function or hematologic function. Patients who were unable to remain prone for half an hour and patients with a known contrast medium allergy were ineligible.

\section{Treatment regimen}

Eligible patients were randomly assigned to neoadjuvant chemotherapy with docetaxel and epirubicin (NACT group), or to neoadjuvant chemotherapy combining rh-endostatin with docetaxel and epirubicin (NACT+rh-endostatin group), followed by final surgery. Patients in the NACT group received 3 cycles of docetaxel and epirubicin (docetaxel $75 \mathrm{mg} / \mathrm{m}^{2}$, IV on day 1 of each 21-day cycle; epirubicin $60 \mathrm{mg} / \mathrm{m}^{2}$, IV on day 1 of each 21-day cycle). Patients in the $\mathrm{NACT}+$ rh-endostatin group received 3 cycles of rh-endostatin plus docetaxel and epirubicin (docetaxel $75 \mathrm{mg} / \mathrm{m}^{2}$, IV on day 1 of each 21 -day cycle; epirubicin $60 \mathrm{mg} / \mathrm{m}^{2}$, IV on day 1 of each 21-day cycle; rh-endostatin $7.5 \mathrm{mg} / \mathrm{m}^{2}$, IV on $1^{\text {st }}$ to $14^{\text {th }}$ day of each 21-day cycle).

\section{DCE-MRI}

All patients were evaluated twice by DCE-MRI. One was performed within one week prior to the first cycle of chemotherapy and the other was performed after the completion of the planned treatment but before surgery. The radiologist who performed the DCE-MRI examinations was blind to the clinical outcome of the patients. The DCE-MRI examination of both breasts was carried out with a 3.0T scanner (MAGNETOM Trio, SIEMENS, German) in the radiology department of Xijing hospital. Patients were examined in the prone position with a dedicated phased array breast-surface coil. Routine magnetic imaging for morphology of the breast was first acquired using $\mathrm{T}_{2}$ - tirm in the transverse and sagittal planes (repetition time $=6490 \mathrm{msec}$, echo time $=60$ msec, flip angle $=120^{\circ}$, slice thickness $=4 \mathrm{~mm}$, interslice gap $=0.4 \mathrm{~mm}$, acquisition matrix $=320 \times 320$ ). The field of view was adapted to fit the size of the breasts, ranging from $210 \times 200 \mathrm{~mm}$ to $310 \times 200 \mathrm{~mm}$. A non-enhanced, fat-suppressed, three-dimensional fast low-angle shot $\mathrm{T}_{1}$ sequence was acquired in the transverse plane (repetition time $=7 \mathrm{msec}$, echo time $=3 \mathrm{msec}_{,}$, slice thickness $=1 \mathrm{~mm}$, interslice gap $=0.2$ $\mathrm{mm}$, acquisition matrix $=450 \times 300$, flip angle $=15^{\circ}$ ), which covered the entire breasts. Next, the dynamic study was performed in the transverse plane using a three-dimensional fast low-angle shot, as described previously, five times 20 seconds after the bolus injection of gadolinium diethylene-triamine pentaacetic acid (Magnevist, Schering, Berlin, Germany).
Gd-DTPA was infused in the antecubital vein at a dosage of $0.1 \mathrm{mmol} / \mathrm{kg}$ body weight by a power injector at a speed of $2 \mathrm{ml} / \mathrm{sec}$ and followed by a $20 \mathrm{ml}$ saline flush at the same speed.

\section{Image analysis}

The acquired MR images were analyzed by two double-blind radiologists with extensive breast MRI experience. Image subtraction was performed from all series of contrast-enhanced images to improve visualization of areas of contrast uptake and to evaluate their morphologies. Then, the time-signal intensity curve was attained through the Mean Curve program provided by SIEMENS. The subtraction of the basal acquisition from early contrast-enhanced slices was loaded into Image J, version 1.40 (National Institutes of Health, Bethesda, MD, USA), for tumor volume evaluation. The total tumor volume was calculated by summing the tumor area in each section and multiplying by the thickness of each section and expressed in $\mathrm{cm}^{3}$. Tumor response in this study was primarily evaluated by comparing tumor volume, calculated by DCE-MRI, according to the Response Evaluation Criteria in Solid Tumors[15]. The signal enhancement ratio (SER) compares early to late enhancement in the lesions to reflcet the rate of contrast washout in the tumor. SER is calculated by $S E R=(S 1-S 0) /(S 2-S 1)$, where $S 0$ is the baseline signal intensity, $S 1$ is the signal measured at 90 seconds, where $S 2$ is the MRI signal intensity at 270 seconds after contrast delivery.

A pharmacokinetic, two-compartment, bidirectional exchange model was used for pharmacokinetic analysis of the images to evaluate the vasculature permeability and perfusion of the tumor. A fixed-T1, fuzzy c-means method, which combines a fixed-T1, fuzzy c-means technique with a reference region model and removes the need for measuring the arterial input function or baseline $\mathrm{T} 1$ or $\mathrm{T} 1(0)$, was used to obtain two main pharmacokinetic parameters[16]. These two parameters were $\mathrm{K}^{\text {trans }}$ (forward transfer constant from the vascular space to the tumors), which primarily reflects the wash-in of contrast agents, and $v_{e}$ (extravascular extracellular volume fraction), which primarily represents the overall leakage space of the tumor. The data were processed with Matlab (The MathWorks, Natick, MA).

\section{Immunohistochemistry}

All patients underwent surgery within one week after the final DCE-MRI examination, at which point the surgical specimens were available for immunohistochemistry. Hematoxylin and eosin staining and Ki-67 and CD-34 expression detection were performed on formalin-fixed and paraffin-embedded 
surgical specimens as described previously[17]. Two experienced breast pathologists who were blind to the original clinical and histological diagnoses interpreted the immunohistochemical slides. Any CD34-stained endothelial cell or endothelial cell cluster that was clearly separated from adjacent microvessels, tumor cells, and connective tissues was considered to be a single countable microvessel[18]. Microvessel density measurements were performed by counting the number of $\mathrm{CD} 34+$ microvessels in three high-power $(400 \times)$ fields randomly selected within a section.

\section{Statistical analyses}

The results of the parameters including tumor sizes $\left(\mathrm{V}_{\mathrm{t}}\right)$, SER, $\mathrm{K}^{\text {trans }}$ and $\mathrm{V}_{\mathrm{e}}$ and MVD were tested for normality with Kolmogorov-Smirnov test. The tumor sizes $\left(\mathrm{V}_{\mathrm{t}}\right), \mathrm{K}^{\text {trans }}$ and $\mathrm{v}_{\mathrm{e}}$ acquired by DCE-MRI before and after treatments were compared using Student's t-tests. MVD and the changes $\left(\Delta \mathrm{V}_{\mathrm{t}}, \Delta \mathrm{K}^{\text {trans, }}\right.$, and $\left.\Delta \mathrm{V}_{\mathrm{e}}\right)$ within each patient were calculated and compared between the two groups using Student's t-tests too. The measure of agreement between tumor size measured by DCE-MRI and histopathology was tested used Kappa test. The correlations between the MVD and DCE-MRI parameters, including SER, Ktrans and $\mathrm{v}_{\mathrm{e}}$ were analyzed using Pearson correlations. All statistical analyses were performed with SPSS version 16.0 (SPSS Inc., Chicago, IL, USA). The results with P values of less than 0.05 were considered statistically significant.

\section{Results}

\section{Patient characteristics}

6 of the 70 patients enrolled in this study failed to complete the 3-cycle chemotherapy ( 1 case due to economic reasons, 1 case due to uncontrollable hyperglycemia, 2 patients requested surgery before the completion of chemotherapy, and 2 patients refused to continue chemotherapy). Therefore, a total of 64 patients completed all 3 cycles of chemotherapy. 31 were in the NACT group and 33 were in the $\mathrm{NACT}+$ rh-endostatin group.

\section{Tumor growth inhibition induced by NACT and NACT+rh-endostatin treatment}

Among the 31 patients in the NACT group, DCE-MRI identified 2 CR (6.5\%), 19 PR (61.3\%), 9 SD $(29.0 \%)$ and 1 PD (3.2\%) subjects. The rate of objective clinical response (CR/PR) was $67.7 \%(21 / 31)$. Mean tumor size $\left(\mathrm{V}_{\mathrm{t}}\right)$ measured by DCE-MRI decreased from $28.53 \pm 10.62 \mathrm{~cm}^{3}$ at baseline to $13.58 \pm 6.35 \mathrm{~cm}^{3}$. The mean change in tumor size was $15.95 \pm 4.32 \mathrm{~cm}^{3}$, and tumor growth inhibition was achieved in $67.7 \%$ patients $(21 / 31)$. The Ki-67 proliferation index was $32.79 \pm 10.25 \%$ after final surgery (Figure $1 \mathrm{~A}$ ). In contrast, among the 33 patients in the NACT+rh-endostatin group, DCE-MRI identified 5 CR (15.2\%), 25 PR (75.7\%), 1 SD (3.0\%) and 2 PD $(6.1 \%)$ subjects. The rate of objective clinical response (CR/PR) was $90.9 \%(30 / 33)$, which was higher than that in the NACT group $(\mathrm{P}=0.021)$. After $\mathrm{NACT}+$ rh-endostatin treatment, mean tumor size $\left(\mathrm{V}_{\mathrm{t}}\right)$ decreased from $31.52 \pm 11.58 \mathrm{~cm}^{3}$ at baseline to $10.34 \pm 4.36 \mathrm{~cm}^{3}$. The mean change in tumor size was $21.18 \pm 7.32 \mathrm{~cm}^{3}$, which was greater than that of the NACT group $(67.2 \%$ vs. $55.9 \%, \mathrm{P}=0.000)$ (Table 1$)$. Tumor growth inhibition was achieved in $66.7 \%$ patients (22/33). The Ki-67 proliferation index was $12.47 \pm 5.11 \%$ after final surgery, which was much lower than in the NACT group $(\mathrm{P}=0.000)$ (Table 2 and Figure 1B). These results suggest that rh-endostatin synergized the anti-tumor effect of docetaxel and epirubicin-based chemotherapy in locally advanced breast cancer.

Table I. Comparison of histopathological response of breast cancers to different chemotherapeutic regimens.

\begin{tabular}{|c|c|c|c|}
\hline \multirow{2}{*}{$\begin{array}{l}\text { Tumor size } \\
\left(\mathrm{mm}^{3}\right)\end{array}$} & \multicolumn{2}{|l|}{ Groups } & \multirow[t]{2}{*}{$p$ value } \\
\hline & NACT $(n=31)$ & $\begin{array}{l}\text { NACT+rh-endostatin } \\
(\mathrm{n}=33)\end{array}$ & \\
\hline Pre-treatment & $28.53 \pm 10.62$ & $31.52 \pm 11.58$ & 0.742 \\
\hline Post-treatment & $13.58 \pm 6.35$ & $10.34 \pm 4.36$ & 0.013 \\
\hline Changes & $15.95 \pm 4.32$ & $21.18 \pm 7.32$ & 0.000 \\
\hline
\end{tabular}

Table 2. The responses of microvessel to different chemotherapeutic regimen in breast cancers.

\begin{tabular}{llll}
\hline Parameters & Groups & \multirow{2}{*}{$p$ value } \\
\cline { 2 - 3 } & NACT $(\mathrm{n}=29)$ & $\begin{array}{l}\text { NACT+rh-endostatin } \\
(\mathrm{n}=28)\end{array}$ & \\
\hline $\mathrm{K}^{\text {trans }}\left(\mathrm{min}^{-1}\right)$ & & & 0.891 \\
Pre-treatment & $0.51 \pm 0.16$ & $0.57 \pm 0.18$ & 0.032 \\
Post-treatment & $0.25 \pm 0.12$ & $0.20 \pm 0.08$ & 0.026 \\
Changes & $0.26 \pm 0.07$ & $0.37 \pm 0.11$ & \\
Ve & & & 0.935 \\
Pre-treatment & $0.74 \pm 0.18$ & $0.71 \pm 0.24$ & 0.021 \\
Post-treatment & $0.46 \pm 0.16$ & $0.34 \pm 0.15$ & 0.023 \\
Changes & $0.28 \pm 0.09$ & $0.37 \pm 0.12$ & 0.827 \\
SER (\% ) & & & 0.042 \\
Pre-treatment & $216.28 \pm 83.56$ & $237.28 \pm 78.56$ & 0.018 \\
Post-treatment & $112.75 \pm 36.35$ & $85.28 \pm 21.94$ & \\
Changes & $103.93 \pm 24.18$ & $152.61 \pm 28.37$ & 0.000 \\
MVD & & & 0.000 \\
Post-treatment & $36.05 \pm 9.64$ & $18.67 \pm 6.53$ & \\
Ki-67 proliferation index $(\%)$ & & $12.47 \pm 5.11 \%$ & \\
Post-treatment & $32.79 \pm 10.25 \%$ &
\end{tabular}




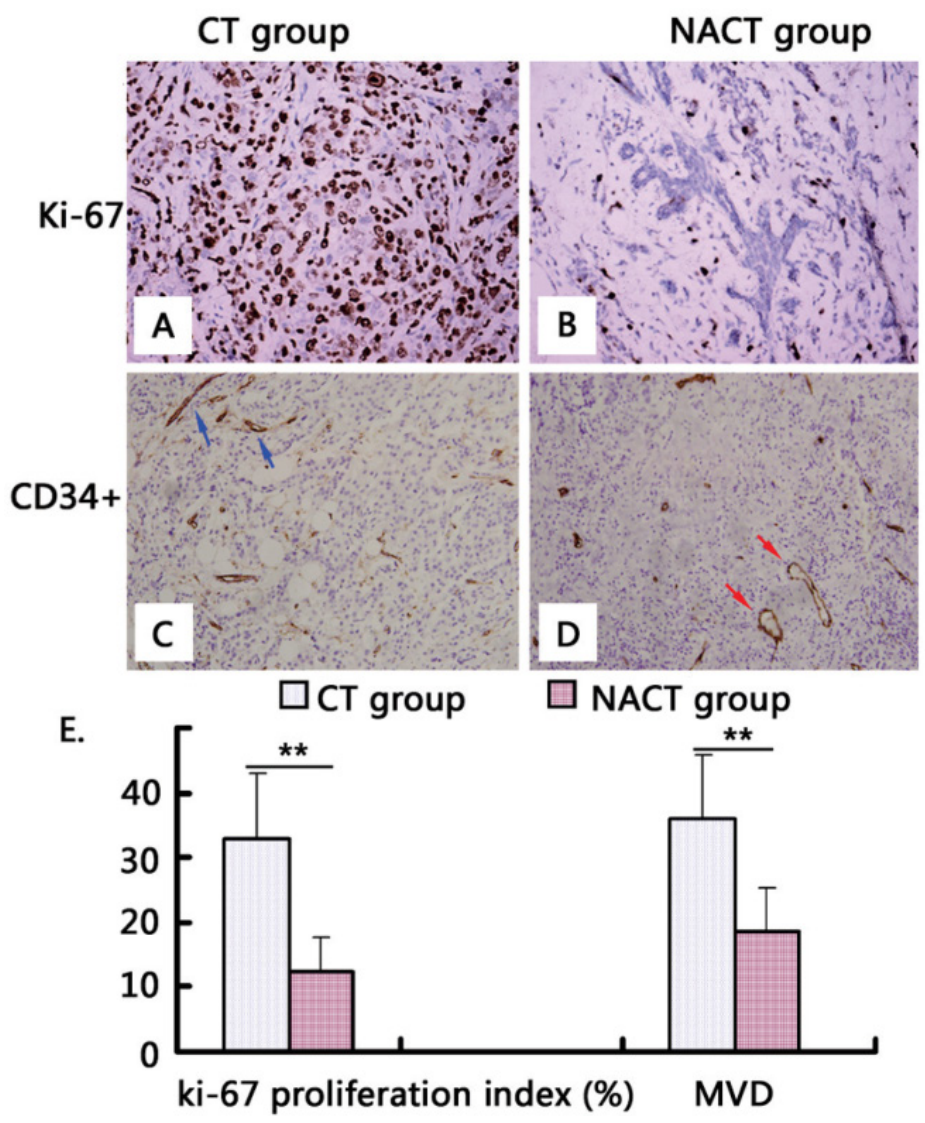

Figure I. Immunohistochemistry of locally advanced breast cancers excised after the completion of different chemotherapeutic regimens. Panels A and B show representative fields of Ki-67 staining in breast cancers in the NACT and NACT+rh-endostatin groups, respectively. Panels $C$ and D showed the representative fields of CD34 immunostaining. The blue arrows indicate irregularly distributed microvessels with giant branches in breast cancers receiving NACT treatment while the red arrows indicate more mature microvessels in breast cancers receiving NACT+rh-endostatin treatment. Panel E shows the quantitative comparisons of the Ki-67 proliferation index and MVD in the center of breast cancers in the NACT and NACT+rh-endostatin groups. **, significant at $\mathrm{P}<0.0 \mathrm{I}$.

The mean tumor size measured by histopathology after surgery was $15.62 \pm 7.45 \mathrm{~cm}^{3}$ in the NACT group and was bigger than that measured by DCE-MRI. The measure of agreement Kappa value was 0.778 . The mean tumor size measured by histopathology after surgery was $12.53 \pm 5.72 \mathrm{~cm}^{3}$ in the $\mathrm{NACT}+$ rh-endostatin group and was bigger than that measured by DCE-MRI too. The measure of agreement Kappa value between them was 0.752 .

\section{Angiogenesis inhibited by NACT+rh-endostatin treatment}

Morphological study revealed a more mature tumor vasculature in the NACT+rh-endostatin group than in the NACT group. It was shown that microvessels in the NACT group were more irregularly distributed and had larger and more numerous branches compared with those in the NACT+rh-endostatin group (Figure $1 \mathrm{C}$-D). In addition, MVD visualized by specific anti-CD34 antibody in tumors treated with $\mathrm{NACT}+$ rh-endostatin was markedly lower than that in the NACT group $(18.67 \pm 6.53$ vs. $36.05 \pm 9.64, \mathrm{P}=0.000)$ (Figure $1 \mathrm{E}$ ).

The DCE-MRI study showed that signals from the breast cancer and involved axillary lymph nodes were quickly and significantly enhanced after the injection of contrast agent in both groups (Figure 2A-D, 3A-D). The steep time-signal intensity curve suggested that contrast agents wash-in and wash-out the tumor very quickly following contrast injection (Figure 2D). 3D-maximum intensity projection (3D-MIP) of MRI T1-weighted images post-injection of a low-molecular contrast agent highlighted the irregular boundary of the tumor with marked enhancement and neovascularization around the tumor (Figure 2E).The tumor and the swelling left axillary lymph node involved shrank after NACT (Figure 2F, G). After chemotherapy, there was a platform appeared in the time-signal intensity curve when the signal of the tumor reach a peak (Figure 2H, I). 3D-MIP manifested the mild reduction of the tumor and neovascularity after NACT(Figure 2J).The swelling left axillary lymph node involved shrank significantly 
after NACT+rh-endostatin (Figure 3B, G). The slope of time-signal intensity curve of the tumor decreased significantly $(1.9 \pm 0.7$ vs. $3.5 \pm 1.4, \mathrm{P}=0.000)$ (Figure 3D, E, I, J). 3D-MIP of MRI T1-weighted images showed the obvious reduction of the tumor volume and angiogenesis (Figure $3 \mathrm{C}, \mathrm{H}$ ).

Quantitative analysis of DCE-MRI data showed that there was no difference in tumor SER, $\mathrm{V}_{\mathrm{e}}$ or $\mathrm{K}^{\text {tran }}$ between the two groups prior to treatment. It was also shown that after NACT treatment, SER of breast cancers decreased significantly from $216.28 \pm 83.56 \%$ to $112.75 \pm 36.35 \%$, $\mathrm{K}^{\text {trans }}$ decreased from $0.51 \pm 0.16 \mathrm{~min}^{-1}$ at baseline to $0.25 \pm 0.13 \mathrm{~min}^{-1}$ and $\mathrm{v}_{\mathrm{e}}$ decreased from $0.74 \pm 0.18$ at baseline to $0.46 \pm 0.12$. In summary, the reductions in tumor SER, $\mathrm{v}_{\mathrm{e}}$ and $\mathrm{K}^{\text {trans }}$ were $48 \%, 39 \%$, and $51 \%$, respectively, in the NACT group. In contrast, after NACT+rh-endostatin treatment, the SER of breast cancers decreased significantly from $237.28 \pm 78.56 \%$ to $85.28 \pm 21.94 \%$, $\mathrm{K}^{\text {trans }}$ decreased from $0.57 \pm 0.18 \mathrm{~min}^{-1}$ to $0.20 \pm 0.08 \mathrm{~min}^{-1}$, and $\mathrm{v}_{\mathrm{e}}$ decreased from $0.71 \pm 0.24$ at baseline to $0.34 \pm 0.15$. In summary, the reductions in tumor SER, $\mathrm{v}_{\mathrm{e}}$ and $\mathrm{K}^{\text {trans }}$ were $64 \%$, $67 \%$, and 52\%, respectively, in the $\mathrm{NACT}+$ rh-endostatin group. It was noted that reductions in SER ( $64 \%$ vs. $48 \%, \mathrm{P}=0.18)$ and $\mathrm{K}^{\text {trans }}(67 \%$ vs. $39 \%, \mathrm{P}=0.26)$ were statistically significantly greater in the NACT+rh-endostatin group than in the NACT group (Table 2). These results demonstrate that rh-endostatin can exert an anti-angiogenesis effect in locally advanced breast cancer.

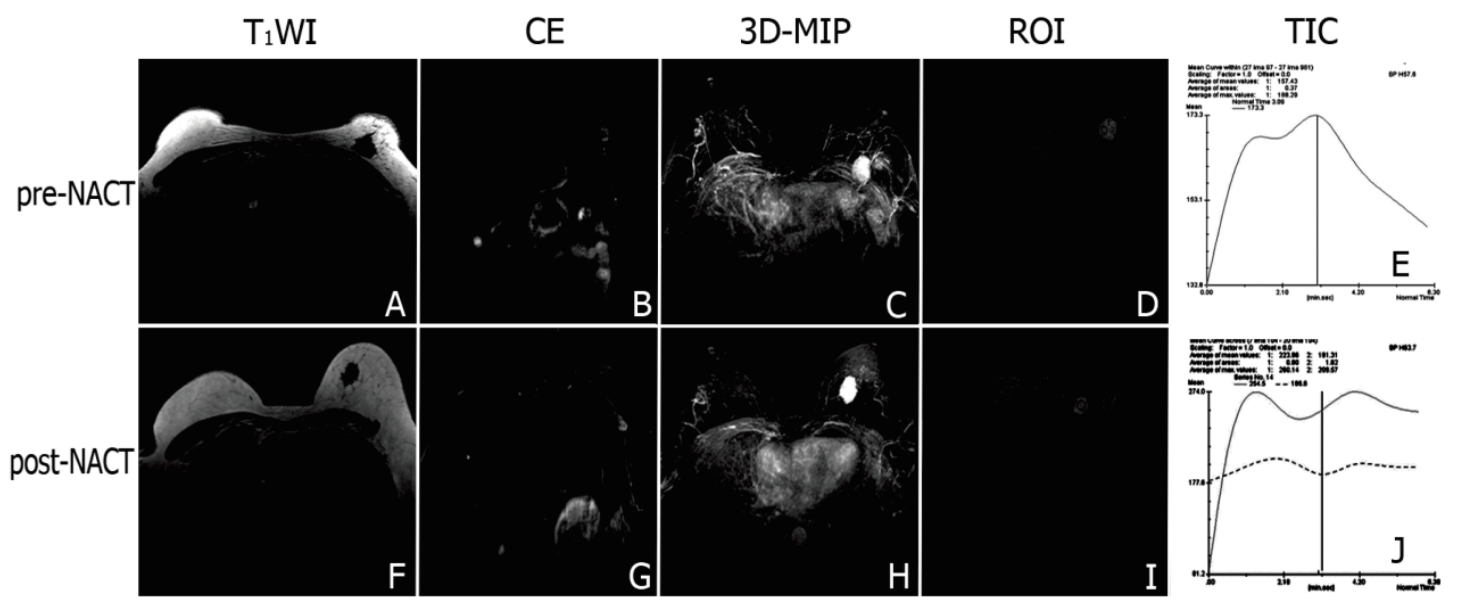

Figure 2. Representative DCE-MR images of locally advanced breast cancers before and after NACT. Signal of the tumor and the swelling left axillary lymph node involved were markedly enhanced after contrast agent injected (A-B). 3D-MIP of contrast-enhanced TI-weighted images highlighted the irregular boundary of the tumor with marked enhancement and neovascularization around the tumor (C). The steep time-signal intensity curve suggested that contrast agents wash-in and wash-out the tumor quickly (D, E). After chemotherapy, the tumor and the swelling left axillary lymph node involved shrank $(F, G)$, and the neovascularity reduced mildly $(H)$. There was a platform appeared in the time-signal intensity curve when the signal of the tumor reached a peak $(I, J)$.

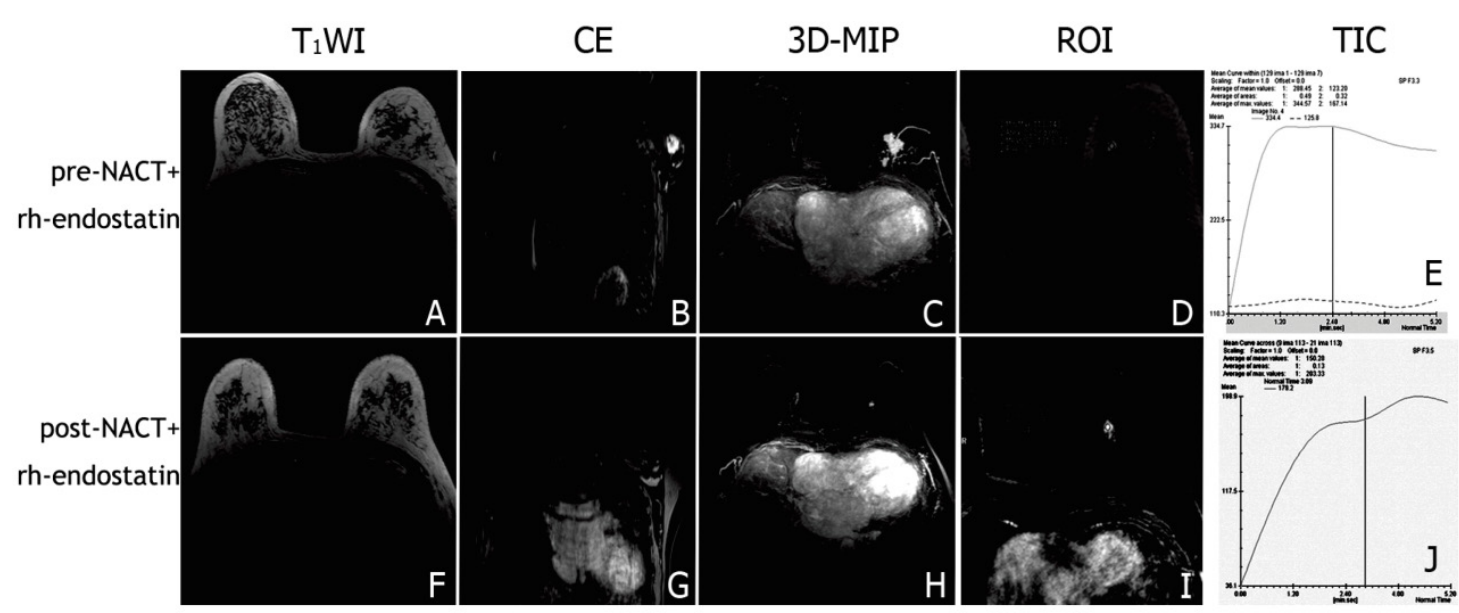

Figure 3. Representative DCE-MR images of locally advanced breast cancers before and after NACT+rh-endostatin treatment. The tumor was difficult to be differentiated from the normal gland tissue (A, F). The swelling left axillary lymph node involved shrank significantly after NACT+rh-endostatin (B, G). 3D-MIP of contrast-enhaced TI-weighted images showed the obvious reduction of the tumor volume and angiogenesis. $(C, H)$. The slope of time-signal intensity curve of the tumor decreased significantly (D, E, I, J). 

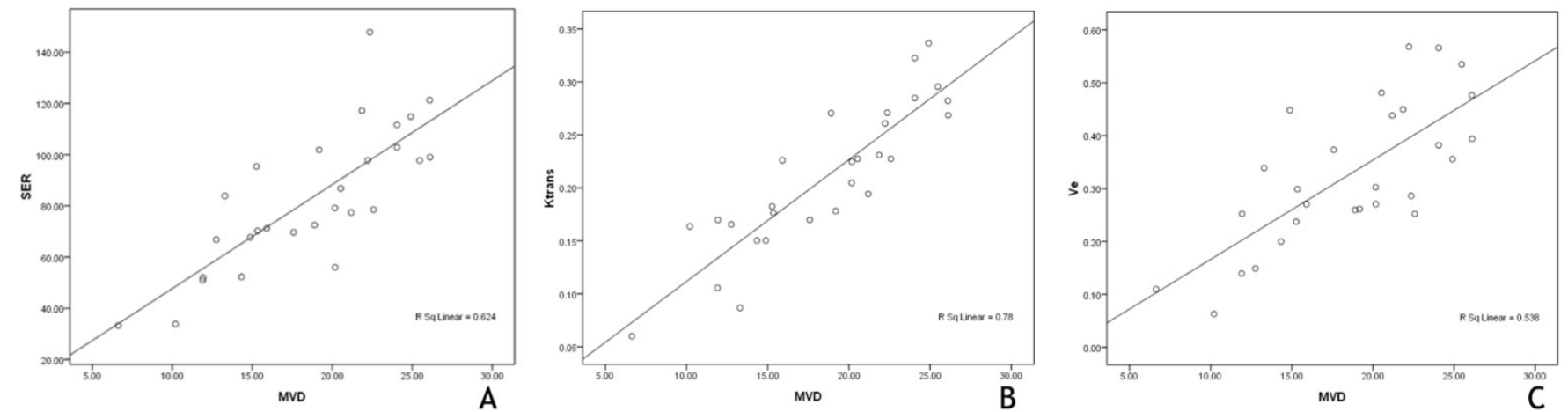

Figure 4. The correlation plots of MVD with DCE-MRI parameters. The MVD values obtained from the immunohistochemical study were significantly correlated with the tumor SER $(r=0.79, P=0.00)(A), K$ trans $(r=0.88, P=0.00)(B)$, and $v_{e}(r=0.73, P=0.00)(C)$, which were obtained from the second DCE-MRI evaluation after the completion of 3-cycle NACT+rh-endostatin treatment.

\section{Correlation of MVD with DCE-MRI parame- ters}

Our study showed that the MVD values obtained from the immunohistochemical study were significantly correlated with the tumor SER $(\mathrm{r}=0.79, \mathrm{P}$ $=0.00), \mathrm{K}^{\text {trans }}(\mathrm{r}=0.88, \mathrm{P}=0.00)$, and $\mathrm{V}_{\mathrm{e}}(\mathrm{r}=0.73, \mathrm{P}=$ 0.00 ), which were obtained from the second DCE-MRI evaluation after the completion of 3-cycle $\mathrm{NACT}+$ rh-endostatin treatment (Figure 4A-C). These results suggest that DCE-MRI can be used as a proxy biomarker of MVD in locally advanced breast cancer.

\section{Discussion}

Excessive proliferation of tumor vessels caused by over-expressed pro-angiogenic factors and down-regulated angiogenesis inhibitors is the histopathological basis for the growth and metastasis of malignant tumors[19]. Therefore, anti-angiogenesis is a promising therapeutic strategy to treat malignant disease by starving tumors of a blood supply. Rh-endostatin is one of the new generations of angiogenesis inhibitor[20]. Besides its inherent anti-angiogenic capability on endothelial cells, rh-endostatin may also play a direct cytotoxic role in inducing programmed cell death in cancer cells[21]. At least in lung cancer cells, it has been shown that endostatin can reduce intracellular expression of the anti-apoptotic proteins $\mathrm{Bcl}-2$ and $\mathrm{Bcl}-\mathrm{XL}$ and induce the activation of caspase- 3 , which initiates the process culminating in apoptosis[22]. These results suggest that regulations of angiogenesis and tumor cell death are not always independent. Conventional cytotoxic chemotherapy with docetaxel and epirubicin may induce cell death in breast cancer cells, leading to tumor shrinkage and improvement of clinical symptoms. Likewise, the formation of new neoplastic ves- sels may also be reduced[23]. Such cross regulation of the two physiological processes could also be observed in neoadjuvant+ rh-endostatin-treated breast cancers, which includes quickly decreasing intrinsic tumor vasculatures, gradually developing mature arteries, and synergistically strengthening inhibition of tumor growth and expansion. The above-mentioned findings were confirmed by both DCE-MRI and MVD assessments in our present study. The underlying mechanisms of action of rh-endostatin on angiogenesis and tumor cell death are not fully elaborated although some studies note that key angiogenic molecules, such as VEGF and VEGFR, could exhibit direct inhibition of tumor cell survival signals and result in apoptosis[7].

In our study, we emphasized $K^{\text {trans }}$ and $V_{t}$, which are acquired from DCE-MRI assessment, in the evaluation of anti-angiogenic efficacies of different chemotherapeutic regimens and the response of tumors to them. Because $\mathrm{K}^{\text {trans }}$ represents not only tumor perfusion over a unit volume but also tumor vascular permeability, it is usually used as an index of angiogenesis status in cancers[24]. The present study indicates that $\mathrm{K}^{\text {trans }}$ value of the tumor is consistent with MVD; this finding was in accordance with previous studies in which broad correlations between various MRI variables and tumor microvascular density have been shown[25]. Given that the enhancement kinetic values of each pixel that covers the entire tumor are statistically analyzed in the DCE-MRI assessment, $K^{\text {trans }}$ is actually a mean value of the angiogenic characters of the whole neoplasm[26]. Our study reveals a moderate to severe reduction of $K^{\text {trans }}$ in locally advanced breast cancers, depending on the therapeutic regimen accepted. Mean $\mathrm{K}^{\text {trans }}$ was significantly decreased (average 67\%) in breast cancers receiving NACT+rh-endostatin. The decreased $\mathrm{K}^{\text {trans }}$ 
was lower in the breast cancers receiving NACT (average $39 \%$ ), suggesting a much more profound inhibitory effect of rh-endostatin on tumor angiogenesis than conventional cytotoxic chemotherapies. A reduction in $\mathrm{K}^{\text {trans }}$ in tumors exposed to cytotoxic chemotherapy has also been reported by previous studies[27]. It has been shown that docetaxel may have anti-angiogenic activity by reducing the synthesis of pro-angiogenic molecules (such as bFGF and VEGF) in cancer cells[28]. In addition, it was recently discovered that conventional cytotoxic chemotherapy can mobilize endothelial precursor cells, and hence, play an important role in inhibiting neovascularization and tumor growth[29].

According to the Response Evaluation Criteria in Solid Tumors criteria, the anatomic measurement of solid tumors is a well-accepted gold standard for evaluating tumor response to chemotherapy[30]. Significant differences in changes of tumor volume determined by DCE-MRI between the two therapeutic regimens were also indicated in our study, demonstrating an enhanced anti-tumor efficacy of combination chemotherapy with rh-endostatin when compared to conventional cytotoxic chemotherapy alone. In fact, DCE-MRI assessment is much more precise in the determination of tumor size than other techniques, such as histopathology and ultrasound examination, which are not able to discriminate the active neoplastic substance from the necrotic portion of the tumor and thus count all parts of the tumor when determining the tumor size. Theoretically, early Gd-DTPA enhancement in DCE-MRI assessment reflects well-vascularized tumor tissue, while slowly enhanced regions are more likely to reflect necrotic tissue[31]. That is to say, tumor size, as measured by early subtracted DCE-MRI images, is strictly representative of the active residual tumor that is unresponsive to chemotherapy. In contrast, histopathology and ultrasound examination, for example, will recognize the necrotic portion of the tumor as unresponsive active residual tumor and cause an underestimation of tumor response to chemotherapy and create somewhat inaccurate decisions about the correct choice for a successful chemotherapeutic regimen. Briefly, based on the high concordance of DCE-MRI with histopathology in the assessment of tumor shrinkage, we consider the former to have advantages in timeliness and accuracy over other techniques in the evaluation of active residual tumors and tumor response to chemotherapy.

In conclusion, the present study showed pronounced anti-angiogenic and anti-cancer efficacy of neoadjuvant chemotherapy plus rh-endostatin on previously untreated, locally advanced breast cancers.
The findings show a significant improvement over conventional cytotoxic chemotherapy with docetaxel and epirubicin alone. In addition, serial DCE-MRI assessment, which was non-invasive and technically convenient, was introduced into the evaluation of antiangiogenic competence and chemotherapeutic responsiveness of neoadjuvant+rh-endostatin. Moreover, correlation tests revealed high concordance between DCE-MRI assessment and classical histopathological examination, thus providing a rationale for real-time referral of locally advanced breast cancer patients to more optimized individual chemotherapy based on serial DCE-MRI data.

But a number of clinical trials have proved that the effect of antiangiogenetic therapeutics may be transient and not result in impovement of progression free survival or overall survival[32]. There were several limitations in our study including the small population enrolled, the short period observed and inclusion of small tumors for evaluation of tumor response. Therefore we are not clear whether the effect of rh-endostatin would translate into substantial benefit to patients with metastatic breast cancer, even though our study demonstrated significant effect of neoadjuvant chemotherapy plus rh-endostatin on previously untreated, locally advanced breast cancers. It needs further study to make clear whether the addition of rh-endostatin to neoadjuvant chemotherapy could significantly increase the overall survival of metastatic breast cancer as bevacizumab[33].

\section{Acknowledgements}

This work was supported by the National Natural Science Foundation of China (Grant No. 81101091).The authors would like to thank Yinmei Wang for expert technical assistance and Lanlan Ye and Hong Lin for helpful discussion.

\section{Competing Interests}

The authors have declared that no competing interest exists.

\section{References}

1. Breslin TM. Neoadjuvant chemotherapy for breast cancer: a surgeon's checklist. Breast Cancer Res Treat, 2011, 127 (1): 129-131.

2. Specht J, Gralow JR. Neoadjuvant chemotherapy for locally advanced breast cancer. Semin Radiat Oncol, 2009, 19 (4): 222-228.

3. Folkman J. Tumor angiogenesis: therapeutic implications. N Engl J Med, 1971, 285 (21): 1182-1186.

4. LaMontagne KR. Antiangiogenesis: new frontiers in therapeutic development. Angiogenesis, 2005, 8 (4): 285-287.

5. Folkman J. Endogenous angiogenesis inhibitors. APMIS, 2004, 112 (7-8): 496-507.

6. Folkman J. Antiangiogenesis in cancer therapy--endostatin and its mechanisms of action. Exp Cell Res, 2006, 312 (5): 594-607.

7. Ling Y, Yang Y, Lu N, You QD, Wang S, Gao Y, Chen Y, Guo QL. Endostar, a novel recombinant human endostatin, exerts antiangiogenic effect via blocking VEGF-induced tyrosine phosphorylation of 
KDR/Flk-1 of endothelial cells. Biochem Biophys Res Commun, 2007, 361 (1): 79-84.

8. Kumar A, Kumar R, Seenu V, Gupta SD, Chawla M, Malhotra A, Mehta $\mathrm{SN}$. The role of 18F-FDG PET/NACT in evaluation of early response to neoadjuvant chemotherapy in patients with locally advanced breast cancer. Eur Radiol, 2009, 19 (6): 1347-1357.

9. Herbst RS, Hess KR, Tran HT, Tseng JE, Mullani NA, Charnsangavej C, Madden T, Davis DW, McConkey DJ, O'Reilly MS, Ellis LM, Pluda J, Hong WK, Abbruzzese JL. Phase I study of recombinant human endostatin in patients with advanced solid tumors. J Clin Oncol, 2002, 20 (18): 3792-3803.

10. Uzzan B, Nicolas P, Cucherat M, Perret GY. Microvessel density as a prognostic factor in women with breast cancer: a systematic review of the literature and meta-analysis. Cancer Res, 2004, 64 (9): 2941-2955.

11. Chen JH, Bahri S, Mehta RS, Kuzucan A, Yu HJ, Carpenter PM, Feig SA, Lin M, Hsiang DJ, Lane KT, Butler JA, Nalcioglu O, Su MY. Breast cancer: evaluation of response to neoadjuvant chemotherapy with 3.0-T MR imaging. Radiology, 2011, 261 (3): 735-743.

12. Fangberget A, Nilsen LB, Hole KH, Holmen MM, Engebraaten $O$, Naume B, Smith HJ, Olsen DR, Seierstad T. Neoadjuvant chemotherapy in breast cancer-response evaluation and prediction of response to treatment using dynamic contrast-enhanced and diffusion-weighted MR imaging. Eur Radiol, 2011, 21 (6): 1188-1199.

13. Ah-See ML, Makris A, Taylor NJ, Harrison M, Richman PI, Burcombe RJ, Stirling JJ, d'Arcy JA, Collins DJ, Pittam MR, Ravichandran D, Padhani AR. Early changes in functional dynamic magnetic resonance imaging predict for pathologic response to neoadjuvant chemotherapy in primary breast cancer. Clin Cancer Res, 2008, 14 (20): 6580-6589.

14. Ni Q, Ji H, Zhao Z, Fan X, Xu C. Endostar, a modified endostatin inhibits non small cell lung cancer cell in vitro invasion through osteopontin-related mechanism. Eur J Pharmacol, 2009, 614 (1-3): 1-6.

15. Prati R, Minami CA, Gornbein JA, Debruhl N, Chung D, Chang HR. Accuracy of clinical evaluation of locally advanced breast cancer in patients receiving neoadjuvant chemotherapy. Cancer, 2009, 115 (6): 1194-1202.

16. Wilmes LJ, Pallavicini MG, Fleming LM, Gibbs J, Wang D, Li KL, Partridge SC, Henry RG, Shalinsky DR, Hu-Lowe D, Park JW, McShane TM, Lu Y, Brasch RC, Hylton NM. AG-013736, a novel inhibitor of VEGF receptor tyrosine kinases, inhibits breast cancer growth and decreases vascular permeability as detected by dynamic contrast-enhanced magnetic resonance imaging. Magn Reson Imaging, 2007, 25 (3): 319-327.

17. Cho SM, Park YG, Lee JM, Byun JY, Lee KY, Park GS, Kim HS, Lee BY, Lee KH, Song KS. 18F-fluorodeoxyglucose positron emission tomography in patients with recurrent ovarian cancer: in comparison with vascularity, Ki-67, p53, and histologic grade. Eur Radiol, 2007, 17 (2): 409-417.

18. Hansen S, Sorensen FB, Vach W, Grabau DA, Bak M, Rose C. Microvessel density compared with the Chalkley count in a prognostic study of angiogenesis in breast cancer patients. Histopathology, 2004, 44 (5): 428-436.

19. Folkman J. Angiogenesis. Annu Rev Med, 2006, 57: 1-18.

20. Xu W, Ye P, Li Z, Shi J, Wang W, Yao K. Endostar, a recently introduced recombinant human endostatin, inhibits proliferation and migration through regulating growth factors, adhesion factors and inflammatory mediators in choroid-retinal endothelial cells. Mol Biol (Mosk), 2010, 44 (4): 664-670.

21. Liao WJ, Shen $\mathrm{P}, \mathrm{Wu} \mathrm{W}$, Shi M, Luo RC. [Short-term therapeutic effect and safety of endostar combined with XELIRI regimen in the treatment of advanced colorectal cancer]. Nan Fang Yi Ke Da Xue Xue Bao, 2010, 30 (4): 813-814.

22. Zhao C, Tang L, Yin J, Dai X, Zhou G. [Endostar Combined GC in the Treatment of Advanced Non-small Cell Lung Cancer.]. Zhongguo Fei Ai Za Zhi, 2009, 12 (5): 460-462.

23. Petrelli F, Borgonovo K, Cabiddu M, Ghilardi M, Barni S. Neoadjuvant chemotherapy and concomitant trastuzumab in breast cancer: a pooled analysis of two randomized trials. Anticancer Drugs, 2011, 22 (2): 128-135.

24. Hsiang DJ, Yamamoto M, Mehta RS, Su MY, Baick CH, Lane KT, Butler JA. Predicting nodal status using dynamic contrast-enhanced magnetic resonance imaging in patients with locally advanced breast cancer undergoing neoadjuvant chemotherapy with and without sequential trastuzumab. Arch Surg, 2007, 142 (9): 855-861.

25. Nicoletto MO, Nitti D, Pescarini L, Corbetti F, Mencarelli R, Cappetta A, Galligioni A, Pogliani C, Marchet A, Bozza F, Ghiotto C, Griggio L, Zavagno G, Donach ME, Di Maggio C. Correlation between magnetic resonance imaging and histopathological tumor response after neoadjuvant chemotherapy in breast cancer. Tumori, 2008, 94 (4): 481-488.

26. Wu LM, Hu JN, Gu HY, Hua J, Chen J, Xu JR. Can diffusion-weighted MR imaging and contrast-enhanced MR imaging precisely evaluate and predict pathological response to neoadjuvant chemotherapy in patients with breast cancer? Breast Cancer Res Treat, 2012.

27. Uematsu T, Kasami M, Yuen S. Neoadjuvant chemotherapy for breast cancer: correlation between the baseline MR imaging findings and responses to therapy. Eur Radiol, 2010, 20 (10): 2315-2322.

28. Jacot W, Bibeau F, Gourgou-Bourgade S, Gutowski M, Colombo PE, Bleuse JP, Kramar A, Romieu G. Phase II trial of dose dense docetaxel followed by FEC100 as neoadjuvant chemotherapy in patients with operable breast cancer. Am J Clin Oncol, 2010, 33 (6): 544-549.

29. Sano H, Wada S, Eguchi H, Osaki A, Saeki T, Nishiyama M. Quantitative prediction of tumor response to neoadjuvant chemotherapy in breast cancer: novel marker genes and prediction model using the expression levels. Breast Cancer, 2012, 19 (1): 37-45.

30. Costelloe CM, Chuang HH, Madewell JE, Ueno NT. Cancer Response Criteria and Bone Metastases: RECIST 1.1, MDA and PERCIST. J Cancer, 2010, 1: 80-92.

31. Li SP, Makris A, Beresford MJ, Taylor NJ, Ah-See ML, Stirling JJ, d'Arcy JA, Collins DJ, Kozarski R, Padhani AR. Use of dynamic contrast-enhanced MR imaging to predict survival in patients with primary breast cancer undergoing neoadjuvant chemotherapy. Radiology, 2011, 260 (1): 68-78.

32. Kerbel RS. Reappraising antiangiogenic therapy for breast cancer. Breast, 2011, 20 Suppl 3: S56-60.

33. Bear HD, Tang G, Rastogi P, Geyer CE, Jr., Robidoux A, Atkins JN, Baez-Diaz L, Brufsky AM, Mehta RS, Fehrenbacher L, Young JA, Senecal FM, Gaur R, Margolese RG, Adams PT, Gross HM, Costantino JP, Swain SM, Mamounas EP, Wolmark N. Bevacizumab added to neoadjuvant chemotherapy for breast cancer. N Engl J Med, 2012, 366 (4): 310-320. 